\title{
Improved Beamforming for Lateral Oscillations in Elastography Using Synthetic Aperture Imaging
}

Liebgott, Hervé; Basarab, Adrian; Loizeau, Damien; Jensen, Jørgen Arendt; Delachartre, Philippe

Published in:

Proceedings of the EEE Ultrasonics Symposium

Link to article, DOI:

10.1109/ULTSYM.2006.548

Publication date:

2007

Document Version

Publisher's PDF, also known as Version of record

Link back to DTU Orbit

Citation (APA):

Liebgott, H., Basarab, A., Loizeau, D., Jensen, J. A., \& Delachartre, P. (2007). Improved Beamforming for Lateral Oscillations in Elastography Using Synthetic Aperture Imaging. In Proceedings of the EEE Ultrasonics Symposium (pp. 2168-2171). IEEE. https://doi.org/10.1109/ULTSYM.2006.548

\section{General rights}

Copyright and moral rights for the publications made accessible in the public portal are retained by the authors and/or other copyright owners and it is a condition of accessing publications that users recognise and abide by the legal requirements associated with these rights.

- Users may download and print one copy of any publication from the public portal for the purpose of private study or research.

- You may not further distribute the material or use it for any profit-making activity or commercial gain

- You may freely distribute the URL identifying the publication in the public portal 


\title{
Improved beamforming for lateral oscillations in elastography using synthetic aperture imaging
}

\author{
H. Liebgott ${ }^{1}$, A. Basarab ${ }^{1}$, D. Loizeau ${ }^{1}$, J.E. Wilhjelm ${ }^{2}$, J.A. Jensen ${ }^{3}$, P. Delachartre ${ }^{1}$ \\ ${ }^{1}$ CREATIS, CNRS UMR 5515, \\ INSERM U630, Université Claude \\ Bernard Lyon 1, INSA Lyon \\ Lyon, France \\ e-mail: name@creatis.insa-lyon.fr \\ ${ }^{2}$ Center for Arteriosclerosis Detection \\ With Ultrasound \\ Technical University of Denmark \\ Kgs. Lyngby, Denmark \\ e-mail: jw@oersted.dtu.dk \\ ${ }^{3}$ Center for Fast Ultrasound Imaging \\ Technical University of Denmark \\ Kgs. Lyngby, Denmark \\ e-mail: jaj@oersted.dtu.dk
}

\begin{abstract}
In this paper we present a beamforming technique based on synthetic aperture imaging that enables to improve the radio-frequency (RF) ultrasound images with lateral oscillations for lateral displacement estimation. As described in previous work, in order to increase the accuracy of the lateral displacement estimation using images with lateral oscillations, it is necessary to reduce both the wavelength of the lateral oscillations and the width of the point spread function (PSF). This is reached in this work, by doing emit and receive beamforming using synthetic aperture data. We show that the wavelength of the lateral oscillations can be reduced by a factor 2, and the width of the PSF can be reduced by a factor $\sqrt{2}$. We have used the images obtained by this beamforming technique for lateral displacement estimation in the field of elastography. We show that with this new approach it is possible to improve the lateral displacement estimation by nearly $25 \%$ by reducing the standard deviation of the error of the lateral estimation.
\end{abstract}

Keywords: synthetic aperture imaging, lateral oscillations, elastograhy, beamforming, displacement estimation

\section{INTRODUCTION}

Within tissue elasticity imaging with ultrasound, our team has previously developed a method that enables us to estimate axial and lateral components of the displacement field between two ultrasound RF images using phase information [1]. We have shown how important it is to optimize the way the RF image is generated, in order to obtain better estimation results. Like in the method developed by Jensen et.al, for blood flow imaging [2] we use a PSF that oscillates in both directions of the image. This PSF is generated by using a particular beamformer; a plane wave is emitted, and the lateral profile of the PSF is controlled by receive beamforming only. Quadratic dynamic focusing is used together with a dynamic transducer apodization function featuring two peaks.

In this work we assume a spatially separable PSF in the axial and lateral dimensions of the RF image. The lateral profile of the PSF showing oscillations is characterized by two parameters: the wavelength of its lateral oscillations $\lambda_{x}$, and the width at half maximum (FWHM) of its Gaussian envelope $\sigma_{\mathrm{x}}$. We have shown in [1] that a decrease of both values would increase the quality of the displacement estimation.
However the values of those parameters are limited by the physical size of the ultrasound transducer. Indeed, a decrease of both parameters leads to a larger active apodization function, which can of course not be larger than the physical size of the probe.

One way to overcome this limitation is to do beamforming both in receive and in emit. In order to have a maximal flexibility we propose in this work to use synthetic aperture imaging. In order to increase the quality of our PSF with lateral oscillations, i.e. decrease both its width and the wavelength of its lateral oscillations, dynamic quadratic focusing is performed in emit and in receive plus an even dynamic apodization function in emit and an odd dynamic apodization function in receive. This method enables to divide by 2 the wavelength of the lateral oscillations and to divide by $\sqrt{2}$ the FWHM of the Gaussian envelope of the PSF. Use of this new PSF with our displacement estimation method enables to increase by nearly $25 \%$ the quality of the lateral displacement estimation compared to the use of a PSF obtained by receive beamforming only.

The paper proceeds as follows. First the principle of synthetic aperture is briefly recalled. Then our beamforming strategy that enables to increase the quality of the PSF is shown. Simulation results showing the improvement in terms of lateral displacement estimation are given. Finally a short conclusion is given.

\section{PrincIPLE OF SYNTHetic APERTURE IMAGING}

\section{A. Data acquisition}

In synthetic aperture imaging, the basic idea is to collect all signals from any transducer element in emit to any transducer element in receive. All beamforming is done in post processing.

In order to do that, each element of the probe is excited separately, and all signals received from all elements are stored in memory. This gives rise to a large amount of data. Typically for a 128 elements probe, $128 \times 128=16284$ signals. 


\section{B. Beamforming possibilities}

In emit, focusing consists basically in applying the right delays in order to have a coherent summation of the contributions coming from all emit elements for one particular point in space called focal point. In receive, focusing consists in applying the right delays to all elements in order to be able to sum signals coming from the same point in space called the focal point.

When doing conventional imaging, the emit focal point is unique. Indeed, once the elements have been excited it is not possible to change the different delays. Whereas in receive focusing it is possible to adapt dynamically the delays, as a function of time to be focused along a given line. With the particular approach of synthetic aperture, it is possible to do dynamic receive and emit focusing. The same statement can be done for apodization, in synthetic aperture, dynamic emit and receive apodization can be achieved.

Let's call $r_{i j}(t)$ the signal received by element $i$, when element $j$ has been emitting. Emit and receive focusing at point $\mathrm{P}$ can be achieved by summing all contributions that can be supposed to have "traveled" from element $\mathrm{j}$ to point $\mathrm{P}$ and back to point $i$. This is done for all pairs of emit and receive elements, and this processing can also be changed for any point in space. All contributions for point $\mathrm{P}$ are denoted $\mathrm{r}_{\mathrm{ij}}(\mathrm{P})$. Finally dynamic emit-receive focusing can be achieved for any point in the image.

In an equivalent way, with synthetic aperture data, it is possible to achieve dynamic emit and receive apodization. Two apodization functions $w_{e}(j, P)$ and $w_{r}(i, P)$, one in emit and one in receive, respectively are defined for one imaging point $\mathrm{P}$ for all emit elements and all receive elements. The apodization and summation is done as follows:

$$
s(P)=\sum_{i} \sum_{j} w_{e}(j, P) w_{r}(i, P) r_{i j}(P)
$$

where $s(\mathrm{P})$ is the pixel of the ultrasound RF final image corresponding to point $\mathrm{P}$.

The choice of the emit and receive apodization functions determine the shape of the lateral profiles of the PSF as described in the following section.

\section{SYNTHETIC APERTURE BEAMFORMING FOR INCREASING PSF RESOLUTION AND LATERAL FREQUENCY}

Here we use the approximation of a separable PSF both spatially and in emit-receive, which leads to:

$$
h(x, z)=h_{e}(x) h_{r}(x) h(z)
$$

$h(z)$ is the axial profile of the PSF, $h_{e}(x)$ and $h_{r}(x)$ are the emit and receive lateral profiles of the PSF. For a given probe, if quadratic focusing is used, it is possible to approximate the lateral PSF profile in emit or in receive to the Fourier transform of the aperture function or apodization function in emit or receive, respectively [3], as given by

$$
F\{w(i)\}=h\left(\frac{x}{\lambda z}\right)
$$

with $\lambda$ the wavelength of the emitted pulse and $z$ the depth of the point of interest.

Here we would like to design the imaging system in order to get a final PSF having a lateral profile showing fast oscillations. In the previous work, the emit lateral profile of the PSF was approximated to be

$$
h_{e}(x)=1
$$

And the receive profile was equal to

$$
h_{r}(x)=\cos \left(2 \pi \frac{x}{\lambda_{x}}\right) \exp \left({ }^{-\pi\left(\frac{x}{\sigma_{x}}\right)^{2}}\right)
$$

In order to increase the frequency of the lateral oscillations, it is possible to use a sin function of same frequency in emit. This will lead to a lateral profile with twice the frequency of the previous one. Indeed if

$$
h_{e}(x)=\sin \left(2 \pi \frac{x}{\lambda_{x}}\right) \exp \left({ }^{-\pi\left(\frac{x}{\sigma_{x}}\right)^{2}}\right)
$$

Then

$$
h(x)=h_{r}(x) h_{e}(x)=\sin \left(2 \pi \frac{x}{\lambda_{x}^{\prime}}\right) \exp \left(^{-\pi\left(\frac{x}{\sigma_{x}^{\prime}}\right)^{2}}\right)
$$

with $\lambda^{\prime}{ }_{x}=\frac{\lambda_{x}}{2}$ and $\sigma_{x}^{\prime}=\frac{\sigma_{x}}{\sqrt{2}}$. In this case both aims are reached, faster oscillations, and thinner PSF.

In order to design the apodization functions that leads to such emit and receive lateral PSF profiles, we use equation (3) obtained under the Fraunhoffer approximation. The emit and receive apodization functions can be exchanged. Here we produce the cos in emit and the sin in receive in order to have only positive coefficients for the emit apodization function.

Using this approximation we obtain the following emit and receive apodization functions:

$$
\begin{gathered}
w_{e / r}=\frac{1}{2}\left(\exp \frac{\left(\frac{x-x_{0}}{\sigma_{0}}\right)^{2}}{+}+\exp ^{\left(\frac{x+x_{0}}{\sigma_{0}}\right)^{2}}\right) \\
\text { with } x_{0}=\frac{\lambda z}{\lambda_{x}} \text { and } \sigma_{0}=\frac{\lambda z \sqrt{2}}{\sigma_{x}}
\end{gathered}
$$

\section{RESUlts}

\section{A. Beamforming validation}

Here we show that using synthetic aperture imaging and a combination of an even apodization function in emit with an odd apodization function in receive, as given in (8), it is possible to divide by a factor 2 the wavelength of the lateral oscillations compared to receive beamforming only.

The simulated probe was a $\mathrm{L} 14-5 \mathrm{~W} / 60$ for use with the Ultrasonix RP system. The PSF is calculated using the Field II program [4]. Figure 1 shows the PSF obtained by receive beamforming only (Figure 1(I)) and by emit and receive beamforming (Figure 1(II)). The emit and receive apodization. 
(I)

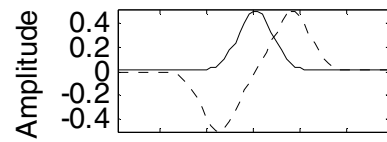

1020304050

(II)

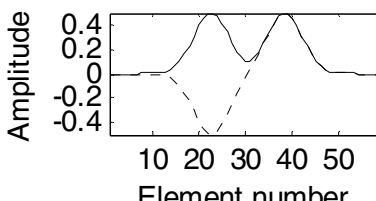

(a)
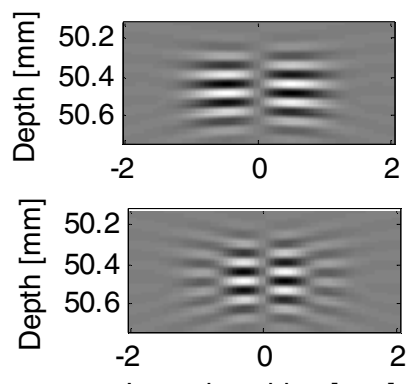

Lateral position [mm]

(b)
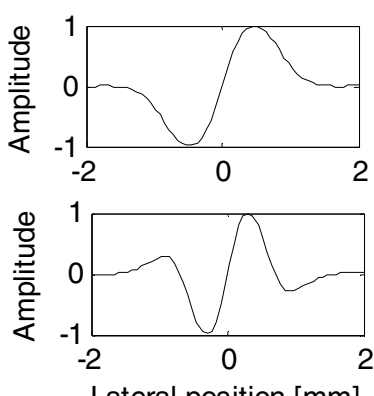

Lateral position $[\mathrm{mm}]$

(c)
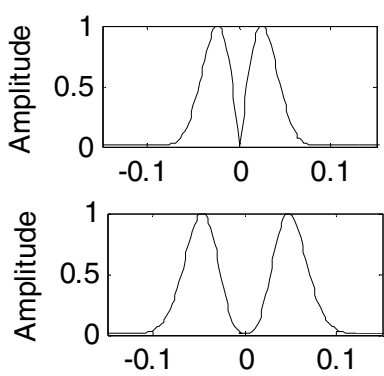

Lateral normalized frequency

(d)

Figure 1: PSF obtained with the Field II program. (I) represents the results for receive beamforming only and (II) for the new approach using emit and receive beamforming. (a) are the emit and receive apodization functions in continuous line and in dotted line, respectively. (b) shows the 2D PSF. (c) shows one lateral profile of the PSF taken at depth 50.5 mm, and (d) the corresponding Fourier transform as a function of normalized spatial frequency.

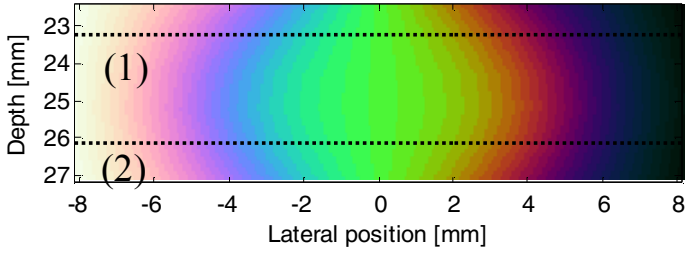

(a)

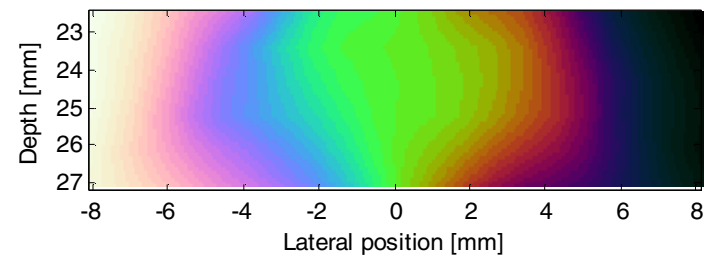

(b)

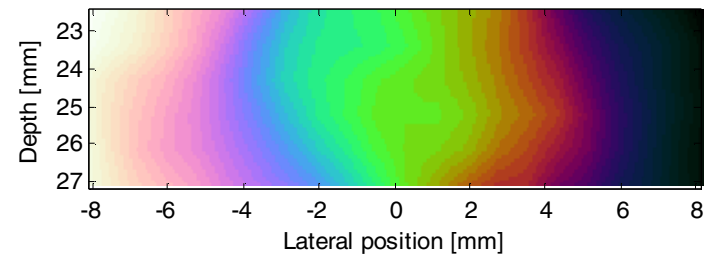

(c)

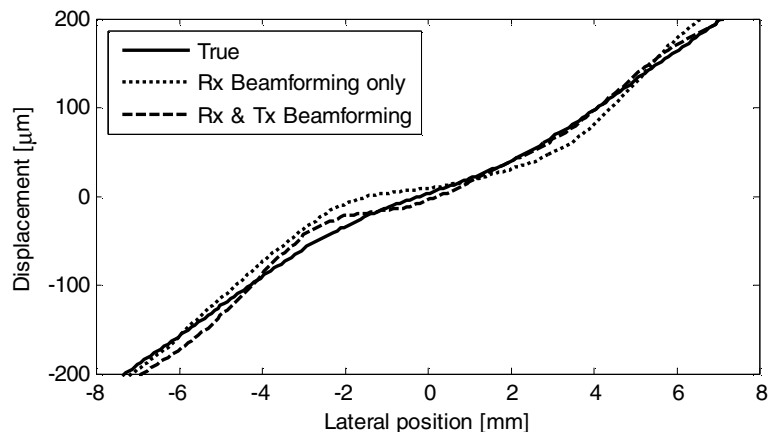

(d) corresponding to depth (1)

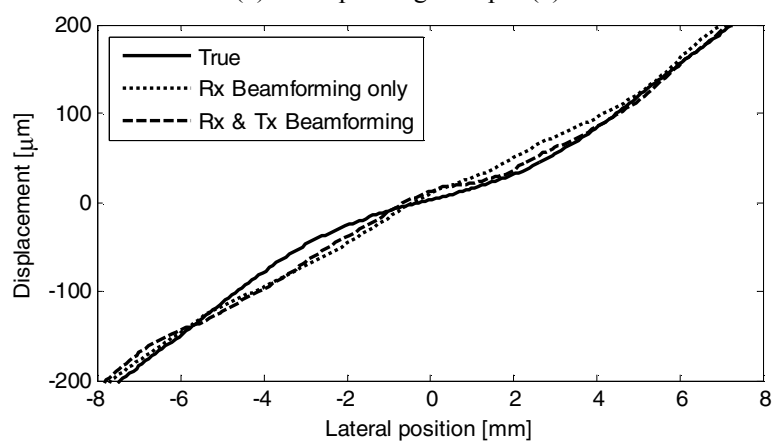

(e) corresponding to depth (2)

Figure 2: True (a) and estimated (b) \& (c) lateral displacement maps, (b) from images obtained by receive beamforming only, (c) from images obtained by emit and receive beamforming. (d) and (e), true and estimated displacement profiles taken at depths indicated (1) and (2) in (a).

functions are given in (a), the 2D PSF are given in (b), (c) gives the lateral profiles of the PSF and (d) the corresponding Fourier transform as a function of normalized lateral frequency

As expected theoretically it is possible to see on this representation that the lateral frequency is doubled thanks to our approach. The expected lateral wavelength for the receive only beamforming case was $2.7 \mathrm{~mm}$ which correspond in our case to a lateral normalized frequency of 0.025 . In the simulation we reach 0.023 . For the new approach a wavelength of $1.35 \mathrm{~mm}$ could be expected which correspond to 0.05 and we obtain 0.045 . Even if the quantitative values do not correspond exactly we have been able to double the lateral frequency which is a very interesting result.
As will be shown in next section using this beamforming technique it is possible to increase the quality of the lateral displacement estimation.

\section{B. Displacement estimation results}

The aim of producing PSF with fast lateral oscillation is to improve the lateral displacement estimation in the field of elastography. In order to show the improvement due to the use of this new image formation scheme, we show some simulation results.

The estimation method that is used here is the one presented in [1]. It is based on the estimation of the zero crossing of the phase of the complex correlation between 1D 
windows taken either from the axial direction of the ultrasound $\mathrm{RF}$ image or from the lateral direction of the image.

A finite element simulation is used to calculate the true displacement inside the medium. Then the RF data are calculated with the simulation program Field II [4].

Figure 2 shows the true and estimated lateral displacement maps obtained from images obtained by receive beamforming only and the ones obtained by receive and emit beamforming. Profiles of the true and estimated displacement are also given. For both profiles represented, the estimation obtained with the images using emit and receive beamforming is closer to the true displacement than the one obtained with images using receive beamforming only. Figure 3 shows the lateral errors histograms for both cases. The lateral error distribution is around $25 \%$ thinner thanks to the new approach. Indeed for the images with receive beamforming only, the standard deviation of the error distribution is equal to $11.4 \mu \mathrm{m}$ whereas for the new approach it is equal to $8.4 \mu \mathrm{m}$.

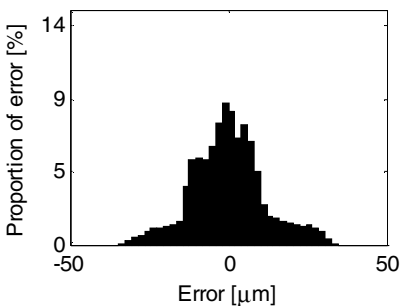

(a)

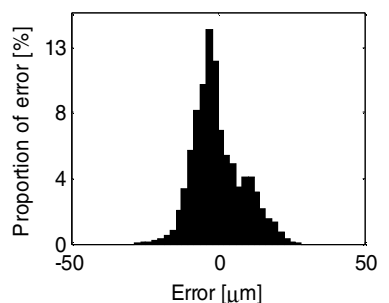

(b)
Figure 3: Lateral estimation error histograms, (a) with images obtained by receive beamforming only, (b) with images obtained by receive and emit beamforming. In (a) the standard deviation of the error distribution is $11.4 \mu \mathrm{m}$, and in (b) it is $8.4 \mu \mathrm{m}$

\section{DISCUSSION}

In this paper we have used synthetic aperture data in order to improve the quality of the ultrasound RF images for lateral displacement estimation in the field of elastography.

One draw back of the previous methods based on lateral oscillations, i.e. only receive beamforming, has been addressed using synthetic aperture data. Using synthetic aperture data, it is possible to do emit and receive beamforming all in postprocessing. This approach enables, by use of an even apodization function in emit and an odd one in receive to double the frequency of the lateral oscillations. The accuracy of the displacement estimation method presented in [1], which was used here, is directly related to the phase slope of the complex correlation between lateral RF signals. Consequently, as this phase slope is proportional to the lateral oscillation's frequency, the new beamforming strategy enables also to improve the quality of the lateral displacement estimation.

In terms of displacement estimation we have been able to increase by $25 \%$ the accuracy of the whole method. Indeed the standard deviation of the error has fallen from $11.4 \mu \mathrm{m}$ to $8.4 \mu \mathrm{m}$.
As a result we can say that the feasibility of our approach has been validated. The next step will be to implement the method on a real scanner. This task should be carried out with the Ultrasonix RP system.

One thing that is important to notice is that there are some new problems that arise and that will have to be addressed which concern the time of acquisition. The methods with lateral oscillations were initially developed for blood flow. In this case the acquisition rate is an important factor because the the blood velocity can be important compared to the velocity of the tissue in the field of elastography. The time of data acquisition is also larger for synthetic aperture data than for conventional ultrasound imaging.

Different solutions could be used, as for example, the ones developed by Jensen and Gran that consist of emitting simultaneously with all elements and separating the signals in post processing before beamforming. The separation of the different signals is achieved thanks to a specific spatial encoding [5].

\section{CONCLUSION}

In this paper we have used a synthetic aperture data acquisition scheme in order to be able to do emit and receive beamforming for adapting images for elastography. The aim was to create faster oscillations in the lateral direction of the ultrasound image than those obtained by receive beamforming only and to decrease the lateral width of the PSF. We have shown that the lateral spatial frequency of the oscillations can be doubled and the width divided by a factor of $\sqrt{2}$ using an even apodization in receive and an odd one in emit.

We have tested our displacement estimation method on synthetic data obtained by simulation. The results show that with our approach it is possible to increase the quality of the estimation. The standard deviation of the error has been reduced by $25 \%$.

The next step is to use this approach on a real phantom using a real scanner. This project should be carried out with the new Ultrasonix RP system.

\section{REFERENCES}

[1] H. Liebgott, J. Fromageau, J. Wilhjelm, D. Vray, and P. Delachartre, "Beamforming scheme for 2D displacement estimation in ultrasound imaging," EURASIP Journal of Applied Signal Processing, vol. 2005, pp. 1212-1220, 2005.

[2] J. A. Jensen and P. Munk, "A new method for estimation of velocity vectors," IEEE Transactions on Ultrasonics, Ferroelectrics and Frequency Control, vol. 45, pp. 837-851, 1998.

[3] J. W. Goodman, Introduction to Fourier optics. New York: McGraw-Hill, 1968.

[4] J. A. Jensen, "Field: A Program for Simulating Ultrasound Systems," Medical \& Biological Engineering \& Computing, vol. 34, pp. 351-353, 1996.

[5] F. Gran and J. A. Jensen, "Multi element synthetic aperture transmission using a frequency division approach," presented at 2003 IEEE Ultrasonics Symposium - Proceedings, Oct 5-8 2003, Honolulu, HI, United States, 2003. 